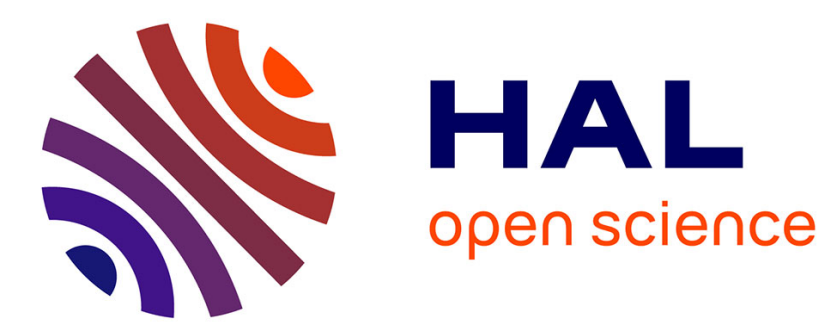

\title{
Origine de la biréfringence naturelle observée dans les cristaux de ZnSe cubique
}

\author{
J. Baillou
}

\section{To cite this version:}

J. Baillou. Origine de la biréfringence naturelle observée dans les cristaux de ZnSe cubique. Revue de Physique Appliquée, 1982, 17 (6), pp.377-381. 10.1051/rphysap:01982001706037700 . jpa-00245009

\section{HAL Id: jpa-00245009 https://hal.science/jpa-00245009}

Submitted on 1 Jan 1982

HAL is a multi-disciplinary open access archive for the deposit and dissemination of scientific research documents, whether they are published or not. The documents may come from teaching and research institutions in France or abroad, or from public or private research centers.
L'archive ouverte pluridisciplinaire HAL, est destinée au dépôt et à la diffusion de documents scientifiques de niveau recherche, publiés ou non, émanant des établissements d'enseignement et de recherche français ou étrangers, des laboratoires publics ou privés. 


\title{
Origine de la biréfringence naturelle observée dans les cristaux de ZnSe cubique
}

\author{
J. Baillou \\ Laboratoire d'Optoélectronique, UER Sciences, 40, avenue du Recteur Pineau, 86022 Poitiers Cedex, France
}

(Reçu le 21 décembre 1981, révisé le 15 mars 1982, accepté le 19 mars 1982)

\begin{abstract}
Résumé. - Les monocristaux de ZnSe préparés par condensation de la phase vapeur cristallisent dans le système de la blende. Cependant ils présentent des défauts de structure qui leur confèrent une faible biréfringence naturelle. Les résultats des mesures relatives à ce phénomène sont exposés, et en les comparant avec ceux obtenus par plusieurs auteurs, deux hypothèses sont proposées sur l'origine et la dispersion de la biréfringence : il apparaît que celle-ci est due à un effet de contrainte d'axe [110] et à un effet de dispersion spatiale, ces deux causes possibles ayant lieu simultanément.
\end{abstract}

\begin{abstract}
ZnSe single crystals prepared by the vapour phase technique grow with the zincblende structure. However these crystals exhibit a weak native birefringence due to structural defects. The results of measurements of this phenomenon are related and compared with the results previously published by several authors. Two assumptions are proposed to explain the origin and the dispersion of the birefringence; it is shown that this later results first from a stress applied along the [110] axis and secondly from spatial dispersion, these both causes can be existing simultaneously.
\end{abstract}

1. Introduction - L'étude de la structure cristalline et des propriétés optiques ou élestrooptiques de cristaux de $\mathrm{ZnSe}$ a été entreprise dans ce Laboratoire depuis plusieurs années. Les monocristaux étudiés sont obtenus à partir d'une méthode de croissance en phase vapeur par sublimation [1]. La caractérisation aux rayons $X$ a révélé la nature cubique faces centrées des différents échantillons [2]. Ceux-ci présentent dans le visible un effet électrooptique linéaire (effet Pockels) relativement important, permettant de penser que $\mathrm{ZnSe}$ est un matériau prometteur pour la réalisation de modulateurs de lumière et d'obturateurs [1, 3]. Toutefois, bien que cristallisant dans le système de la blende, la plupart des échantillons possèdent des défauts de structure qui leur confèrent une anisotropie optique : en l'absence de toute excitation électrique, ils laissent en effet réapparaître entre polariseurs croisés une faibie intensité lumineuse. La biréfringence naturelle issue de ce phénomène parasite a été intensivement étudiée au Laboratoire $[2,4,5]$. A partir des résultats expérimentaux que nous avons obtenus, et par comparaison avec les travaux de différents auteurs [6-9], nous proposons dans cet article deux hypothèses sur l'origine de cette biréfringence.
2. Résultats sur la biréfringence naturelle dans les cristaux de $\mathrm{ZnSe}$. - La biréfringence naturelle a été observée et mesurée sur huit échantillons de $\mathrm{ZnSe}$ provenant de différents lingots et taillés selon les plans (110), (1/10), et (001) de façon qu'ils puissent être ensuite utilisés en effet électrooptique. Le domaine spectral balayé s'étend dans le visible depuis le seuil d'absorption fondamentale jusqu'à $0,6 \mu$. Chaque échantillon a été soumis à deux séries de tests :

1) Recherche des lignes neutres sur les différentes faces du cristal.

2) Mesure de la biréfringence naturelle $\Delta n$ dans les trois directions [110], [110] et [001] par la méthode de De Senarmont.

L'étude expérimentale, détaillée dans deux articles précédents $[2,4]$, a conduit aux résultats suivants :

1) Lorsque la lumière traverse le cristal dans la direction [110], on observe une forte dispersion des lignes neutres.

2) Lorsque la lumière traverse le cristal dans les directions [110] ou [001], on n'observe pas de dispersion des lignes neutres; celles-ci sont confondues avec les arêtes de l'échantillon. 
3) L'intensité de la biréfringence naturelle varie sensiblement avec la longueur d'onde et la direction de propagation de la lumière. Les valeurs obtenues dans les trois directions [110], [110] et [001], notées respectivement $\Delta n_{1}, \Delta n_{2}, \Delta n_{3}$, sont reportées sur la figure 1. Elles représentent typiquement la dispersion

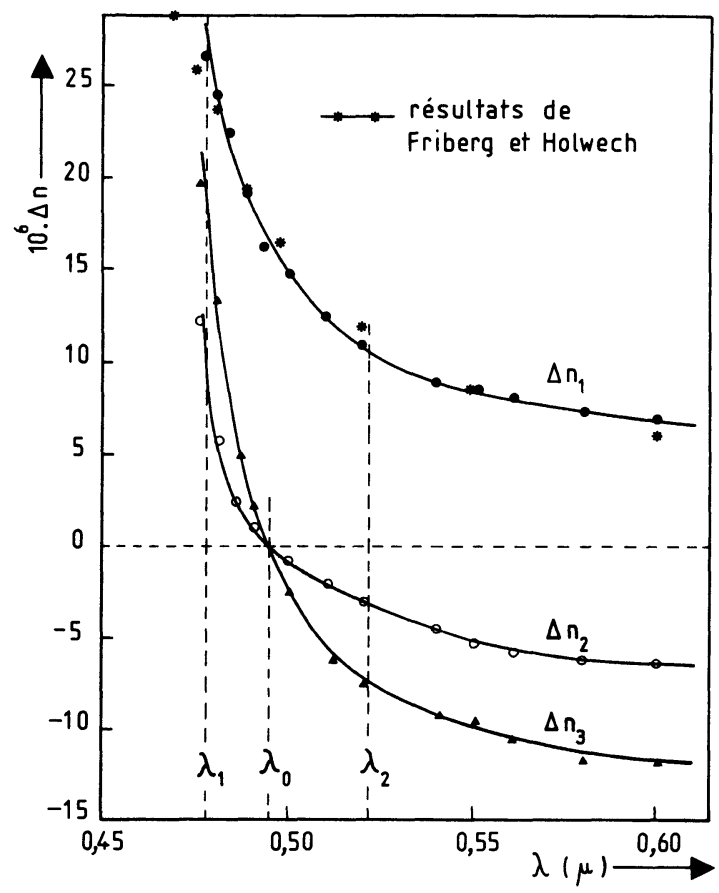

Fig. 1. - Dispersion de la biréfringence naturelle de $\mathrm{ZnSe}$ dans les trois directions de propagation : [110] $\rightarrow \Delta n_{1}$; $[1 \overline{1} 0] \rightarrow \Delta n_{2} ;[001] \rightarrow \Delta n_{3}$.

[Dispersion of the native birefringence of $\mathrm{ZnSe}$ for three directions of propagation : [110] $\rightarrow \Delta n_{1} ;[1 \overline{1} 0] \rightarrow \Delta n_{2}$; [001] $\rightarrow \Delta n_{3}$.

de la biréfringence naturelle dans nos cristaux, les résultats variant peu d'un échantillon à l'autre. On constate sur la figure 1 que la biréfringence $\Delta n_{1}$ ne s'annule jamais dans le spectre étudié, alors que les biréfringences $\Delta n_{2}$ et $\Delta n_{3}$ s'annulent pour une même longueur d'onde, puis changent de signe.

Ces résultats expérimentaux nous ont permis de localiser les axes principaux de l'ellipsoïde des indices dans $\mathrm{ZnSe}$ et de constater la nature biaxiale de la biréfringence naturelle [4]. Il a été montré que l'axe [110] de la blende est un axe de symétrie optique, propriété vérifiée par l'étude cristallographique qui a été menée conjointement $[2,10]$. Nous avons pu ainsi proposer un ellipsoïde des indices relatif à la biréfringence naturelle de $\mathrm{ZnSe}$, dont la représentation est donnée figure 2 .

3. Origine de la biréfringence naturelle - Afin d'expliquer la présence de la biréfringence naturelle dans les cristaux de $\mathrm{ZnSe}$, deux hypothèses peuvent être avancées : des effets de contrainte intervenant en cours de croissance et des effets de dispersion

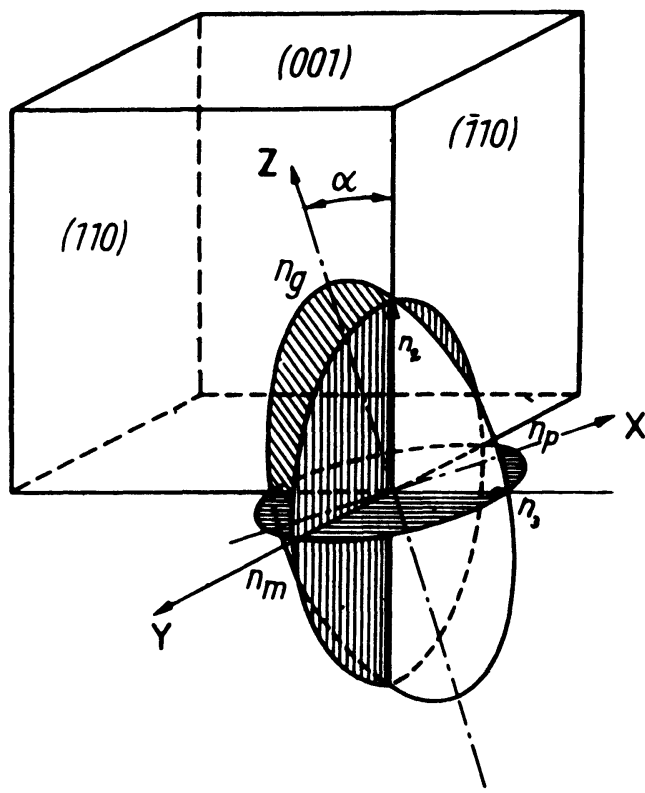

Fig. 2. - Ellipsoïde des indices relatif à la biréfringence naturelle de $\mathrm{ZnSe}$ et ses sections par les 3 plans d'onde (110), (11̄0), (001).

[Sections of the index ellipsoid for the native birefringence of $\mathrm{ZnSe}$ according to the three wave planes (110), (1110), (001).]

spatiale. Nous avons écarté une autre origine possible de la biréfringence, celle due à une succession de phases cubiques et hexagonales dans $\mathrm{ZnSe}$. Plusieurs arguments, détaillés dans une publication antérieure [11], contribuent à éliminer cette dernière hypothèse. Ils ne seront donc pas repris ici.

3.1 Les EFFETS DE CONTRAINTE. - Il est connu que l'application d'une contrainte uniaxiale sur un semiconducteur de type blende modifie sa symétrie eristalline et le rend biréfringent : c'est la piézobiréfringence [7]. Si la contrainte a lieu dans les directions $\langle 100\rangle$ ou $\langle 111\rangle$, le cristal devient optiquement uniaxe, l'axe optique étant orienté ${ }^{-d a n s ~ l a ~ d i r e c t i o n ~}$ de la contrainte. Pour une direction $\langle 110\rangle$ ou arbitraire de la contrainte appliquée, le cristal devient biaxe, l'un des axes de l'ellipsoïde des indices' étant parallèle à l'axe de la contrainte [12].

Les travaux entrepris sur différents matériaux à grand gap ont fait apparaitre une forte dispersion de la piézobiréfringence au voisinage du seuil d'absorption fondamentale $[6,7,13]$. D'après Higginbotham [7], la piézobiréfringence et sa dispersion peuvent être interprétées à partir du schéma des bandes d'énergie du semiconducteur. La structure de bandes de $\mathrm{ZnSe}$ [16] comporte un point singulier à $\mathbf{k}=0$ (seuil d'absorption directe d'énergie $E_{0}$ ) ainsi que d'autres points singuliers dans l'espace des $\mathbf{k}$, pour lesquels la distance interbandes est nettement supérieure à $E_{0}$. Leur influence peut s'interpréter par une énergie moyenne $E_{\mathbf{g}}$ L'application d'une contrainte a pour effet de décomposer le double 
niveau $\Gamma_{8}$ de la bande de valence de $\mathrm{ZnSe}$ à $\mathbf{k}=0$. Il en résulte une modification des fonctions d'onde électroniques et des éléments de matrice intervenant dans le calcul des transitions interbandes. Cette modification entraîne une variation $\Delta \varepsilon_{1}$ de la partie réelle de la constante diélectrique, donc de l'indice de réfraction puisqu'en région de transparence on a $\varepsilon_{1}=n^{2}$.

Pour Yu et Cardona, la contribution du gap $E_{0}$ de $\mathrm{ZnSe}$ est de signe contraire à celle du gap moyen $E_{\mathrm{g}}$. Pour les matériaux à large bande interdite, la contribution de $E_{\mathrm{g}}$ domine aux grandes longueurs d'onde; elle est pratiquement constante pour les matériaux de ce type et elle présente peu de dispersion dans le visible, mais elle est masquée par la contribution de $E_{0}$ au voisinage du seuil fondamental : par suite la dispersion de la piézobiréfringence s'accompagne d'un changement de signe.

Ce modèle théorique a été vérifié expérimentalement sur ZnSe par Yu et Cardona [6] : leurs résultats sont reproduits sur la figure 3 . On observe une forte dispersion de la piézobiréfringence en limite du seuil d'absorption fondamentale, puis un changement de

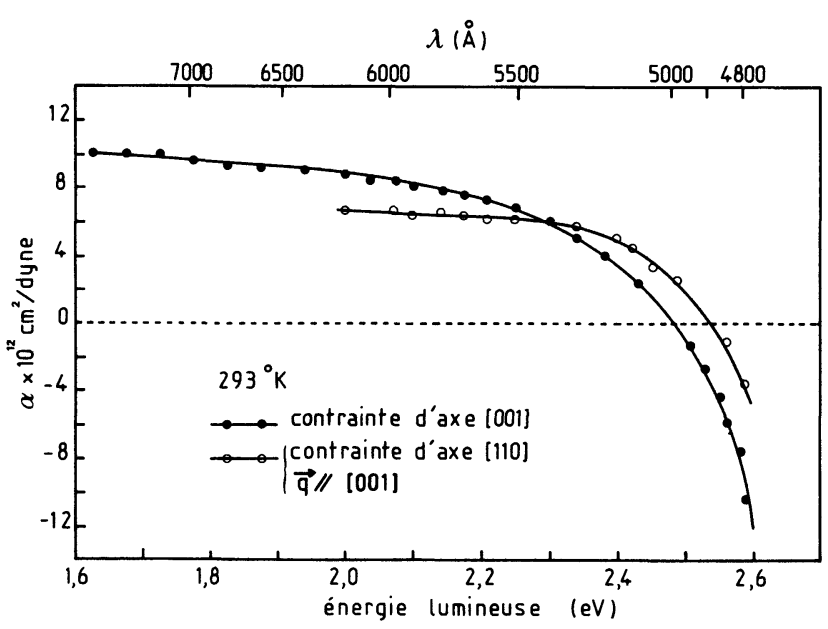

Fig. 3. - Résultats de Yu et Cardona sur le coefficient de piézobiréfringence de $\mathrm{ZnSe}$ mesuré à la température ambiante [6].

[Results of $\mathrm{Yu}$ and Cardona on the piezobirefringence coefficient measured at room temperature [6].]

signe et une dispersion beaucoup plus faible aux grandes longueurs d'onde, que ce soit pour une contrainte $\sigma$ d'axe [001] ou d'axe [110] et une direction de propagation [001] de la lumière. Dans ce deuxième cas, le changement de signe du coefficient de piézobiréfringence $\alpha=\Delta \varepsilon_{1} / \sigma$ a lieu vers $4900 \AA$.

3. 2 LES EFFETS DE DISPERSION SPATIALE. - L'anisotropie optique des cristaux cubiques peut aussi résulter du phénomène de dispersion spatiale. La théorie macroscopique, détaillée par Agranovich et Ginzburg [9], a été vérifiée sur Si par Pastrnak et Vedam [14] et sur GaAs par Yu et Cardona [15].
La relation générale entre la composante $D_{i}$ de l'induction électrique et la composante $E_{j}$ du champ électrique d'une onde électromagnétique traversant un milieu diélectrique s'écrit :

$$
D_{i}(\omega, \mathbf{q})=\varepsilon_{i j}(\omega, \mathbf{q}) E_{j}(\omega, \mathbf{q})
$$

q étant le vecteur d'onde électromagnétique, les indices $i$ et $j$ permutant de 1 à 3 . Le tenseur de terme général $\varepsilon_{i j}$ peut donc varier avec $\omega$ (c'est la dispersion temporelle) et avec q (c'est la dispersion spatiale). L'intensité de celle-ci est caractérisée par le paramètre $a / \lambda$, où $a$ est une dimension caractéristique de l'ordre de grandeur d'une maille cristalline et $\lambda$ la longueur d'onde électromagnétique. On néglige souvent la dispersion spatiale lorsque la longueur d'onde est grande vis-à-vis des dimensions des systèmes atomiques, ce qui est le cas dans le visible : dans un cristal, $a / \lambda$ est de l'ordre de $5 \AA / 5000 \AA=10^{-3}$.

Etant donné la faiblesse de la dispersion spatiale, on utilise un développement limité pour le tenseur $\varepsilon_{i j}$ et le tenseur inverse :

$$
\left.\begin{array}{rl}
\varepsilon_{i j}(\omega, \mathbf{q}) & =\varepsilon_{i j}(\omega, 0)+i \gamma_{i j l}(\omega) q_{l}+\alpha_{i j l m}(\omega) q_{l} q_{m} \\
\varepsilon_{i j}^{-1}(\omega, \mathbf{q}) & =\varepsilon_{i j}^{-1}(\omega, 0)+i \delta_{i j l}(\omega) q_{l}+\beta_{i j l m}(\omega) q_{l} q_{m}
\end{array}\right\}
$$

Pour les cristaux cubiques non centrosymétriques tels que $\mathrm{ZnSe}$, les tenseurs $\gamma_{i j l}$ et $\delta_{i j l}$ sont nuls. Si l'on néglige les termes du second ordre en $q$, les expressions $\varepsilon_{i j}(\omega, \mathbf{q})$ et $\varepsilon_{i j}^{-1}(\omega, \mathbf{q})$ se réduisent à des scalaires et le milieu est optiquement isotrope. Par contre, si l'on tient compte des termes en $q^{2}$, c'est-à-dire si l'on tient compte de l'orientation du vecteur d'onde $\mathbf{q}$, il est impossible de réduire les termes $\varepsilon_{i j}$ et $\varepsilon_{i j}^{-1}$ à des scalaires et une anisotropie optique apparaît. Agranovich et Ginzburg ont montré que l'utilisation des expressions (2) dans les équations d'onde du milieu diélectrique conduit à des valeurs différentes de l'indice de réfraction suivant la direction de propagation de la lumière. Lorsque le plan d'onde est un plan $\{100\}$ ou $\{111\}$, l'indice de réfraction $n$ est indépendant de l'orientation du vecteur $\mathbf{D}$ contenu dans ce plan; par contre si le plan d'onde est un plan $\{110\}$, la valeur de $n$ dépend essentiellement de la direction du vecteur D. Entre les deux directions orthogonales de $\mathbf{D}\langle 110\rangle$ et $\langle 100\rangle$, il existe une biréfringence maximum, d'intensité :

$$
\begin{aligned}
\Delta n & =(n)_{D\langle 110\rangle}-(n)_{D\langle 100\rangle} \\
& =\frac{1}{2}\left[\beta_{1212}+\frac{1}{2}\left(\beta_{1122}-\beta_{1111}\right)\right] \cdot \frac{n_{0}^{5} \omega^{2}}{c^{2}}
\end{aligned}
$$

$n_{0}$ étant l'indice de réfraction du milieu sans tenir compte de la dispersion spatiale. Cette biréfringence conserve par ailleurs le même signe quand la longueur d'onde varie.

Expérimentalement, une biréfringence résiduelle a été observée dans des cristaux de $\mathrm{ZnSe}$ cubique par 
Friberg et Holwech [8], et entièrement attribuée à la dispersion spatiale. La biréfringence a été mesurée pour une propagation [110] de la lumière. Nous avons reporté leurs résultats sur la figure 1 , afin de pouvoir les comparer aux nôtres (courbe $\Delta n_{1}$ ), obtenus dans des conditions de mesure identiques : on constate un très bon accord entre nos observations et les leurs, dans tout le domaine spectral étudié.

4. Interprétation des résultats obtenus. - Compte tenu des résultats de $\mathrm{Yu}$ et Cardona, Friberg et Holwech, il est possible d'interpréter la plupart de nos résultats expérimentaux sur l'origine et la dispersion de la biréfringence naturelle dans $\mathrm{ZnSe}$, en considérant que les deux causes possibles de cette biréfringence ont lieu simultanément :

1) L'évolution de $\Delta n_{3}$ [001] ne peut s'interpréter que par la piézobiréfringence : si l'on admet que le cristal a subi en cours d'élaboration une contrainte d'axe [110], notre résultat sur $\Delta n_{3}$ (Fig. 1) est en bon accord avec celui de $\mathrm{Yu}$ et Cardona (Fig. 3). En particulier nous avons obtenu un changement de signe pour une longueur d'onde comprise entre $4850 \AA$ et $4950 \AA$ suivant les échantillons, alors que ces auteurs ont noté une inversion de signe de la piézobiréfringence à $4900 \AA$ pour une contrainte d'axe [110] et une direction de propagation [001] de la lumière. D'autre part la dispersion de $\Delta n_{3}$ est nettement plus faible quand on s'éloigne du seuil d'absorption fondamentale, ce qui vérifie également le résultat théorique.

2) L'évolution de $\Delta n_{1}$ [110] peut être attribuée à la dispersion spatiale, car notre résultat figure 1 est tout à fait identique à celui de Friberg et Holwech. Il est possible cependant que l'application d'une contrainte [110] rendant le cristal biaxe, on puisse avoir simultanément dans cette direction un effet de piézobiréfringence, mais cet effet serait masqué par la dispersion spatiale, étant donné la grande similitude de nos résultats avec ceux de Friberg et Holwech, et compte tenu du fait que nous n'avons pas observé de changement de signe pour $\Delta n_{1}$.

3) Les effets de dispersion spatiale et de piézobiréfringence peuvent $a$ priori avoir lieu simultanément dans la direction [110], mais comme dans cette direction on a observé une évolution de $\Delta n_{2}$ assez comparable à celle de $\Delta n_{3}$, en particulier avec un changement de signe à la même longueur d'onde, il semble qu'ici l'effet de contrainte soit prépondérant. Toutefois, la présence simultanée de la dispersion spatiale dans la direction [1 $1 \overline{1} 0]$ et son absence dans la direction [001] peuvent être à l'origine de l'évolution légèrement différente des deux courbes $\Delta n_{2}$ et $\Delta n_{3}$ aux grandes longueurs d'onde.

4) Si l'on admet l'existence d'une contrainte [110] sur le cristal de $\mathrm{ZnSe}$, celui-ci est alors devenu optiquement biaxe, et l'un des axes de l'indicatrice doit être parallèle à la direction [110]. Cette propriété se trouve confirmée par les conclusions que nous avons tirées de nos résultats expérimentaux :

a) Nous avons en effet établi que l'axe [110] était l'un des axes principaux de l'ellipsoïde des indices de $\mathrm{ZnSe}$ (Fig. 2).

b) A partir des mesures de $\Delta n_{1}, \Delta n_{2}$ et $\Delta n_{3}$, nous avons mis en évidence la dispersion des axes optiques de $\mathrm{ZnSe}$, propriété caractéristique d'un cristal biaxe [4].

c) Nous avons constaté par ailleurs la nature biaxiale de la biréfringence naturelle à partir de la rotation des lignes neutres du cristal dans la direction de propagation [110] et de leur immobilité dans les directions [11̄0] et [001].

5. Origine et calcul de la contrainte responsable de la piézobiréfringence. - $\mathrm{Si}$ la dispersion spatiale peut s'expliquer par les effets quadrupolaires qui se manifestent dans la direction [110], pour expliquer la présence de piézobiréfringence nous pensons qu'au cours de son élaboration par croissance à partir de la phase vapeur, le lingot de $\mathrm{ZnSe}$ a été soumis à une contrainte dirigée suivant l'axe [110] et intervenant lors de la phase de refroidissement.

Cette contrainte thermique aurait pour origine le gradient de température existant entre les deux extrémités du lingot au cours du refroidissement, car il semble exclu qu'elle soit attribuée à l'ampoule de quartz dans laquelle le $\mathrm{ZnSe}$ est élaboré à $1270^{\circ} \mathrm{C}$, le coefficient de dilatation thermique du séléniure de zinc étant nettement supérieur à celui du quartz [10].

Les résultats théoriques sur la photoélasticité des cristaux cubiques permettent d'évaluer l'intensité de cette contrainte. En effet, la biréfringence d'un cristal appartenant à la classe de symétrie $\overline{4} 3 \mathrm{~m}$ et soumis à une contrainte uniaxiale $\sigma$ [001] s'écrit, pour une direction d'observation orthogonale à celle de la contrainte [12],

$$
\Delta n=\frac{1}{2} n_{0}^{3}\left(\pi_{11}-\pi_{12}\right) \sigma .
$$

Pour une contrainte d'axe [111], le calcul donne :

$$
\Delta n=\frac{1}{2} n_{0}^{3} \pi_{44} \sigma .
$$

Les coefficients $\pi_{i j}$ étant les coefficients piézooptiques du cristal. Dans le cas de $\mathrm{ZnSe}$, ceux-ci ont pour valeur $[17,18,19]$ :

$$
\begin{aligned}
& \pi_{11}=-1,45 \times 10^{-7} \mathrm{~cm}^{2} / \mathrm{kg} \\
& \pi_{12}=0,22 \times 10^{-7} \mathrm{~cm}^{2} / \mathrm{kg} \\
& \pi_{44}=-1,22 \times 10^{-7} \mathrm{~cm}^{2} / \mathrm{kg} .
\end{aligned}
$$

Par ailleurs, des mesures effectuées sur $\mathrm{ZnSe}$ par Dubenskii et al. [20] ont montré que si la contrainte appliquée au cristal est d'axe [110] et la direction d'observation [001], l'évolution de $\Delta n / \sigma$ en fonction de la longueur d'onde est sensiblement la même que dans le cas où la contrainte est d'axe [111]. A partir de nos résultats sur $\Delta n_{3}[001]$, il est par conséquent 
possible d'établir l'ordre de grandeur de la contrainte [110] correspondante : pour $\lambda=5500 \AA$, la mesure de la biréfringence naturelle a donné $\Delta n_{3}=-10^{-5}$ (Fig. 1); à cette biréfringence est donc associée la contrainte $\sigma[110]$ d'intensité :

$$
\sigma_{[110]}=\frac{2 \Delta n_{3}}{n_{0}^{3} \pi_{44}} \simeq 8,8 \mathrm{~kg} / \mathrm{cm}^{2}
$$

en prenant $n_{0}=2,65$ à $5500 \AA$ [21].

Cette valeur est du même ordre de grandeur que le résultat obtenu par Kulakovskii et al. [22] ( $\sigma$ de 10 à $20 \mathrm{~kg} / \mathrm{cm}^{2}$ ); elle est de plus tout à fait compatible avec les calculs effectués récemment par Daunay et al. [23] sur la contrainte susceptible de se développer pendant la synthèse du cristal de $\mathrm{ZnSe}$ ( $\sigma$ de l'ordre de quelques dizaines de $\mathrm{kg} / \mathrm{cm}^{2}$ ).
6. Conclusion - Les différentes comparaisons entre nos résultats expérimentaux et les travaux de plusieurs auteurs nous ont amenés à proposer deux hypothèses sur l'origine et la dispersion de la biréfringence naturelle dans les cristaux de $\mathrm{ZnSe}$ cubique : il apparaît que cette biréfringence est due à un effet de contrainte d'origine thermique et d'axe [110], associé à un effet de dispersion spatiale, chaque phénomène étant plus ou moins intense selon la direction de propagation de la lumière. Ces deux hypothèses justifient en effet assez bien les courbes de dispersion d'indices de la figure 1 ainsi que la nature biaxiale de la biréfringence naturelle observée. En utilisant les coefficients piézooptiques du cristal, nous avons déduit de nos mesures une valeur de la contrainte [110] responsable de la piézobiréfringence; l'ordre de grandeur obtenu est compatible avec les résultats publiés par différents auteurs sur ce sujet.

\section{Bibliographie}

[1] Baillou, J., Lemercier, M., Rivaud, G., Pavis, B., Revue Phys. Appl. 8 (1973) 121.

[2] Daunay, J., Baillou, J., Lemercier, M., DauNay, Jac., Bugnet, P. et Batailler, G., Phys. Status Solidi (a) 37 (1976) 521.

[3] Bugnet, P., Baillou, J., Daunay, J. et Daunay, Jac., Revue Phys. Appl. 14 (1979) 259.

[4] Daunay, J., Revue Phys. Appl. 11 (1976) 739.

[5] BaIllou, J., Thèse de Doctorat ès Sciences Physiques. Université de Poitiers (1981).

[6] Yu, Y. P. et Cardona, M., J. Phys. Chem. Solids 34 (1973) 29.

[7] Higginbotham, C. W., Cardona, M. et Pollack, F. H., Phys. Rev. 184 (1969) 821.

[8] Friberg, A. et Holwech, I., Appl. Phys. 11 (1976) 383.

[9] Agranovich, V. M. et Ginzburg, V. L., Spatial dispersion in crystal optics and the theory of excitons. Interscience publishers (J. Wiley and Sons London) 1966.

[10] Daunay, J., Thèse de Doctorat ès Sciences Physiques. Université de Poitiers (1981).

[11] Baillou, J., Bugnet, P., Daunay, J. et Daunay, Jac., J. Phys. D : Appl. Phys. 12 (1979) 451.
[12] Nye, J. F., Propriétés physiques des cristaux (Dunod, Paris) 1961.

[13] Yu, P. Y., Cardona, M. et Pollack, F. H., Phys. Rev. B 3 (1971) 340.

[14] Pastrnak, J. et Vedam, K., Phys. Rev. B 3 (1971) 2567.

[15] Yu, P. Y. et Cardona, M., Solid State Commun. 9 (1971) 1421.

[16] Cohen, M. L. et Bergstresser, T. K., Phys. Rev. 141 (1966) 789.

[17] Goldstein, L. F., Thompson, J. S., Schroeder, J. B. et Slattery, J. E., Appl. Opt. 14 (1975) 2432.

[18] Berlincourt, D., JafFe, H. et Shiozawa, L. R., Phys. Rev. 129 (1963) 1009.

[19] Adachi, S. et Hamaguchi, C., Phys. Rev. B 19 (1979) 938.

[20] DubenskiI, K. K., Kaplyanskil, A. A. et LozovSKAYA, N. G., Sov. Phys. Solid State 8 (1967) 1644.

[21] Marple, D. T. F., J. Appl. Phys. 30 (1964) 539.

[22] KulakovskiI, V. D. et Kulakov, M. P., Sov. Phys. Crystallogr. 20 (1976) 656.

[23] Daunay, J., Daunay, Jac. et Bugnet, P., à paraître dans The J. Crystal Growth (1982). 